\title{
CONVERSION OF THE FOUR-STRAP ARRAY IN DIII-D TO A TUNABLE TRAVELING WAVE ANTENNA
}

by

D.A. PHELPS, C.P. MOELLER, C.C. PETTY, R.I. PINSKER, P.M. RYAN,* R.G. GOULDING,* and D.J. HOFFMAN*

This is a preprint of a paper presented at the Tenth Topical Conference on Radio Frequency Power in Plasmas, April 1-3, 1993, Boston, Massachusetts, and to be printed in the Proceedings.

Work supported in part by

U.S. Department of Energy Contracts

DE-AC03-89ER51114 and DE-AC05-840R21400

*Oak Ridge National Laboratory

GENERAL ATOMICS PROJECTS 4437 and 3466 APRIL 1993

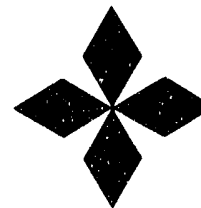

\section{GENERAL

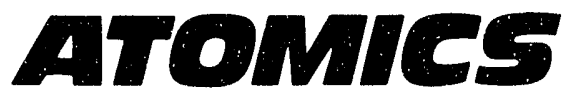




\title{
CONVERSION OF THE FOUR-STRAP ARRAY IN DIII-D TO A TUNABLE TRAVELING WAVE ANTENNA
}

\author{
D.A. Phelps, C.P. Moeller, C.C. PetTy, and R.I. Pinsker \\ General Atomics, San Diego, CA 92186-9784
}

P.M. RYAN, R.H. Goulding, and D.J. HoffmaN

Oak Ridge National Laboratory, Oak Ridge, TN 37830

\begin{abstract}
The in situ conversion of the two-standing wave resonator driven four-strap array in DIII-D to a new type of traveling wave antenna (TWA) is introduced. First observations are reported on (1) broad frequency range tunability; (2) narrow frequency band phase control; (3) single pass transmission loss and modification caused by resistive and reactive sheet simulators of plasma loading; and (4) recirculating power resonance and gain in a traveling wave resonator (TWR) driven TWA.
\end{abstract}

\section{INTRODUCTION}

To maximize fast wave current drive (FWCD) in the ion cyclotron range of frequencies (ICRF), the rf radiation spectrum must track an optimum phase velocity ${ }^{1}$ that increases with electron heating ${ }^{2}$ and changes with $\mathrm{L}-\mathrm{H}$ and other mode transitions, or ELMs. Broadband frequency and phase tunability is needed to access the desired FWCD, electron heating, and ion cyclotron resonance heating scenarios. A transmitter/tuner/antenna system that meets these needs, but also switches rapidly between scenarios, is the ICRF enabling technology challenge for the future.

The combline TWA ${ }^{3-7}$ exhibits phase control between $(0, \pi)$ within a preselected cutoff frequency passband, and thereby suits its principal mission - FWCD at the highest frequency commensurate with transmitter power, FWCD efficiency, and avoidance of ion cyclotron resonance. In-band rapid switching between electron heating during startup, and FWCD during $\mathrm{L}$ and $\mathrm{H}$-mode, is another accessible scenario. ${ }^{8}$ To access ion heating scenarios, however, requires an in-vacuum tunable capacitive load, a change in the tune during a vent, or sets of comblines tuned to preselected frequencies. ${ }^{8}$ This latter solution is viable for next generation tokamaks, such as ITER ${ }^{4}$ and Ignitor. ${ }^{5,6}$ Issues for TWAs in general are the lack of physics and technology validation at high rf power, and/or with plasma loading, sheatb rectification and impurity generation. ${ }^{9}$ To perform near term research on as-built antennas, including broad phase and frequency tunability, consider the externally tuned TWA $^{6}$ described herein.

\section{THE EXTERNALIY TUNED TWR DRIVEN T.WA}

The TWR driven TWA circuit in Fig. 1 utilizes an as-built boxed-in four-strap array with septa and Faraday shield, such as the DIII-D four-strap array mock-up at ORNL (the Mock-up), or the B4C shielded four-strap array now in DIII-D (B4C Prototype). Each strap is made into a transmission line cavity, and tuned for standing wave resonance between the strap-short and an external coax-short using a line stretcher (or tuning capacitor) ${ }^{6,10}$ Power is critically coupled into or out of just the end strapcavities. Interior strap-cavities are powered only by mutual coupling. Power not radiated or absorbed in the walls is recirculated in a TWR, using a $3 \mathrm{~dB}$ hybrid. 
Parameters based on a resolution of theory with experiment are given in Table I. Note that (1) $Z_{0}=42 / 93 \Omega$ implies with/without a Faraday shield (for example); (2) $k_{m n}$ are the TWA coupling factors; (3) $k_{m, m \pm 1}=39 \%$ without septa (as preferred); and (4) $R_{\mathrm{p}}=k_{23} X_{\mathrm{L}} \approx 3 \Omega$ is the critical load resistance (where $k Q=1$ ). Notably, $\mathrm{L}$ and $\mathrm{H}$-mode loads in DIII-D $\mathrm{D}^{11,12}$ are less than this $R_{\mathrm{p}}$ as desired.

Recently, toroidal wave number $\left(k_{\text {tor }}\right)$ spectra for a $1 \mathrm{~m}$ TWA were simulated. Remarkably, nearly $100 \%$ of the power can be absorbed by the plasma without significant change in the $k_{\text {tor }}$ spectrum relative to no absorption, except well above the optimum $k_{\text {tor }}$ for FWCD. Since higher $k_{\text {tor }}$ are more evanescent, ${ }^{11-13}$ there will be much less change in the launched fast wave spectrum.

\section{EXPERIMENTAL RESULTS}

Single-pass observations of the $S$-parameters at TWA ports P1 and P2 were made without the TWR in Fig. 1. The magnitude and phase of reflection $\left(S_{11}, S_{22}\right)$ and transmission $\left(S_{21}, S_{12}\right)$ parameters were measured. Transmission reciprocity was observed. The observed $2 \mathrm{MHz}$ passband was predicted by theory, ${ }^{3}$ using the parameters in Table I. The observed smooth phase variation, about $\pi / 2$ at midband, is consistent with a theory for unidirectional traveling wave (versus a standing wave). By lengthening the strap-cavities, the passband was down-shifted from about 61 to $63 \mathrm{MHz}$ to about 48 to $50 \mathrm{MHz}$, as predicted. ${ }^{3}$ Following this procedure, wider band tuning (e.g., 30 to $120 \mathrm{MHz}$ ) can be achieved.

Frequency responses for the Mock-up are displayed in Fig. 2. Filter-like passbands are predicted by applying combline theory ${ }^{3}$ to a TWA with half-wave resonant strapcavities. ${ }^{6}$ There are no spurious passbands below the first upper filter passband at about $140 \mathrm{MHz}$, as expected. ${ }^{3}$ The highest transmission efficiency occurred for no loading, and is less than unity due to $\sim 10 \%$ reflection from $\mathrm{P} 1$ and $\mathrm{P} 2$, wall absorption, and/or free space radiation. Tuners were not used to reduce these reflections, in order to study TWA passband responses. The family of passband profiles in Fig. 2 are parametric in (a) resistive (carbon filled plastic), or (b) reactive (aluminum) sheet dummy loads of larger area than the Faraday shield. The load was changed by changing the shield-sheet gap. The observed passband attenuation and slight down-shift in Fig. 2(a) compares with expectation for a RLC resonant circuit. ${ }^{6}$ As a potential calibration technique, each external short was replaced by a $2.7 \mathrm{ohm}$ resistor load. The observed attenuation was slightly greater than that for the $1.5 \mathrm{~cm}$ gap. This suggests that plasma relevant 1 to $3 \Omega$ /strap loads ${ }^{11,12}$ can be achieved with plasma relevant 2 to $8 \mathrm{~cm}$ shield-sheet gaps. A family of profiles for shield-metal sheet spacings are shown in Fig. 2(b). The slight increase in frequency with smaller gaps is consistent with reduced antenna inductance caused by the metal sheet. This compares with driven four-strap array measurements, wherein $3 \%$ to $5 \%$ reduction in inductance was observed in the presence of plasma. ${ }^{12}$ These results suggest a recipe for establishing a complex plasma surface impedance ${ }^{6}$ : (1) set the metal wall gap for the right reactance; (2) interstice the resistive sheet to produce the right resistance; and (3) iterate as necessary.

Figure 3 exhibits the transmission passband modifications introduced when the TWA is driven by a TWR, as typified in Fig. 1. At the observed maximum TWR resonance (about $62 \mathrm{MHz}$ ), the roundtrip electrical length at midband (i.e., for $90 \mathrm{deg}$ phasing/strap) was a little under $2 \mathrm{~m}$, which is consistent with prediction. ${ }^{6}$ Furthermore, the observed maximum gain of $1.8: 1$ is consistent with prediction ${ }^{6}$ for a 0.7 roundtrip transmission efficiency and the $3 \mathrm{~dB}$ hybrid coupler. 


\section{CONCLUSIONS AND RECOMMENDATIONS}

The reversible conversion of the B4C prototype in DIII-D to an externally tuned TWA has been discussed. Several critical enabling technologies have been demonstrated:

1. Tunability over 30 to $120 \mathrm{MHz}$ using a line stretcher in each strap cavity.

2. Smooth phase change between $(0, \pi)$ cutoffs within a $2 \mathrm{MHz}$ transmitter bandwidth.

3. Single pass transmission loss and passband modification caused by resistive and reactive sheet simulators of plasma loading.

4. Recirculating power gain in a traveling wave resonator (TWR) driven TWA.

Recommendations for future externally tuned TWA research tasks are:

1. Modify or delete the as-built septa and shields to improve phase control, stability, directionality and coupling to plasma.

2. Demonstrate the in situ conversion of a driven four-strap array to a TWA, as recently demonstrated with the Mock-up. Note in Fig. 4(a) that only two SWR locations need to be shorted. One tuned line inputs power, the other extracts power. A $3 \mathrm{~dB}$ hybrid coupler can be used to recirculate power.

3. Conduct near term $200 \mathrm{~W}$ experiments in DIII-D. Investigate how TWAs respond to plasma transitions and fluctuations in reactive and resistive loading.

Recommendations for alternative TWA concepts are:

1. Develop the present 9 to 27 strap "thin" combline TWA concepts ${ }^{5,6}$ for DIII-D. These comblines can fit in and between ports and tile surface to vessel wall recesses.

2. Study the hybrid TWA concept,$^{13}$ that mixes features of the combline elements, as sketched in Fig. 4(b). This takes advantage of combline enhancements near a preselected frequency for FWCD, while retaining full conventional capability and broadband access to driven FWCD and ion/electron heating scenarios.

3. Design TWAs for two-strap center grounded arrays like those in TFTR and Alcator, and four Jet-type strap arrays like those being fabricated for DIII-D and JET.

\section{ACKNOWLEDGMENT}

This is a report of work sponsored in part by the U.S. Department of Energy under Contract Nos. DE-AC03-89ER51114 and DE-AC05-84OR21400.

\section{REFERENCES}

1. M. Porkolab, Proc. AIP Conf. RF Power in Plasmas 244, p. 197 (1991).

2. J.S. deGrassie et al., Bull. Am. Phys. Soc. 37, 1513 (1992).

3. C.P. Moeller et al., Proc. Euro. Top. Conf. RF Heating (1992) p. 53.

4. D.A. Phelps et al., Bull. Am. Phys. Soc. 37, 1513 (1992).

5. C.P. Moeller et al., 6th Wrkshp. on RF Antenna Design \& Phys., Boulder (1993).

6. D.A. Phelps et al., ibid. (1993).

7. C.P. Moeller, to be presented at this conference (April 1-3, 1993).

8. R.L. Freeman, GA, private communication (December 1992).

9. J. Jacquinot et al., private communication, ibid., Ref. 6 (1993).

10. F. Durodie, 16th Symp. on Fusion Technol., London (1990).

11. M.J. Mayberry et al., to be submitted to Nucl. Fusion, GA-A19565 (1989).

12. R.H. Goulding et al., ibid., Ref. 1 (1991).

13. D.J. Hoffman, ORNL, private communication (March 16, 1993). 


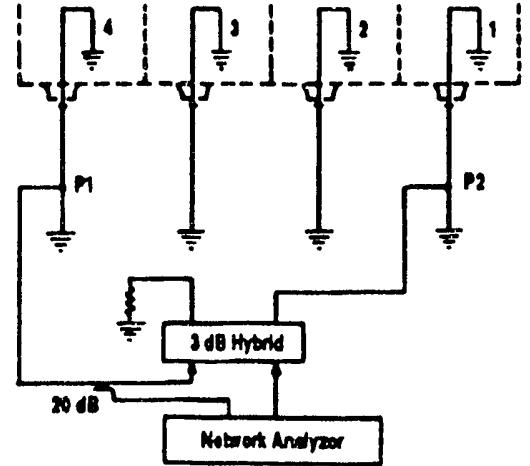

Fig. 1. A TWR driven TWA.
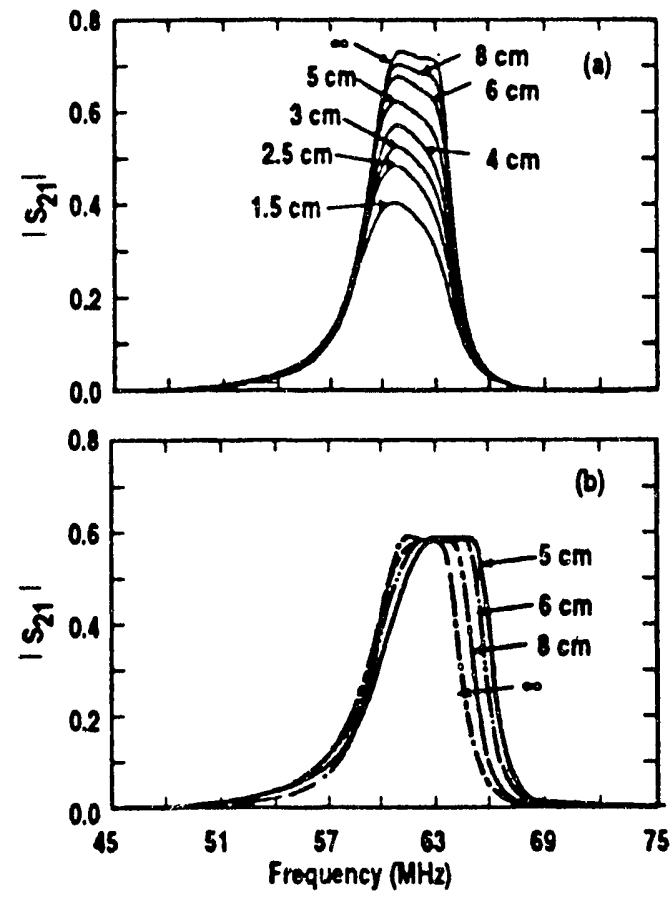

Fig. 2. TWA $\left|S_{21}\right|$ profiles parametric in (a) resistive and (b) reactive sheet loading.

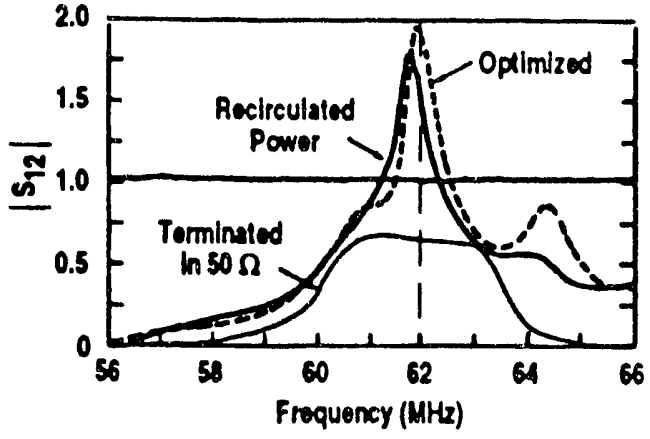

FIG. 3. Observed TWR-TWA circuit gain.

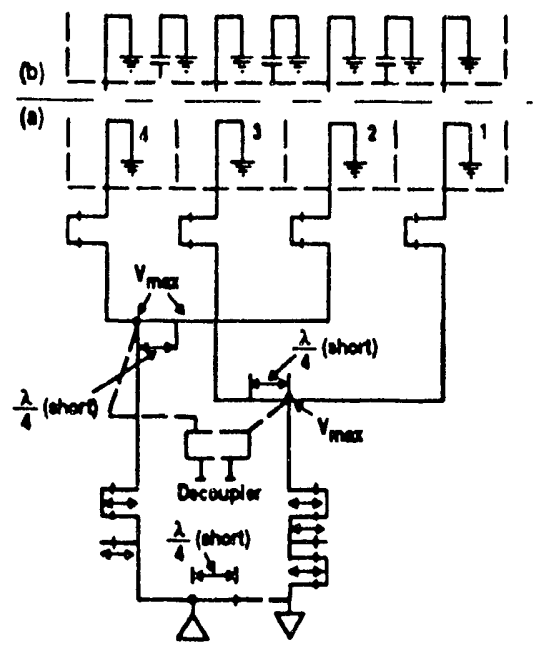

Fig. 4. Conversion of the driven fourstrap array in DIII-D to (a) an externally tuned TWA; (b) a hybrid thereof with combline TWA elements.

Table I. Parameters for the DIII-D Prototype and Mock-up

\begin{tabular}{lcccccccc}
\hline Antenna & $\ell_{\mathrm{p}}(\mathrm{m})$ & $v / c$ & $Z_{0}(\Omega)$ & $R^{\prime}(\Omega / \mathrm{m})$ & $k_{12}=k_{34}$ & $k_{23}$ & $k_{13}=k_{14}$ & $k_{14}(\%)$ \\
\hline Strap Proto. & 0.45 & 0.52 & $42 / 93$ & $-/ 0.015$ & 9.5 & 7.4 & 1.6 & 0.4 \\
Strap Mock-up & 0.45 & 0.52 & $35 / 75$ & $0.12 / 0.015$ & 7.0 & 6.5 & 0.7 & 0.2 \\
\hline Strip Proto. & 0.34 & 1 & 32 & 0.005 & & & \\
Strip Mock-up & 0.38 & 1 & 32 & 0.005 & & & \\
\hline Coax Proto. & $*$ & 1 & 30 & 0.086 & *Strap 1=0.75; $2=0.82 ; 3=0.80 ;$ \\
Coax Mock-up & 0.13 & 1 & 40 & 0.02 & and 4=0.75 m & \\
\hline
\end{tabular}



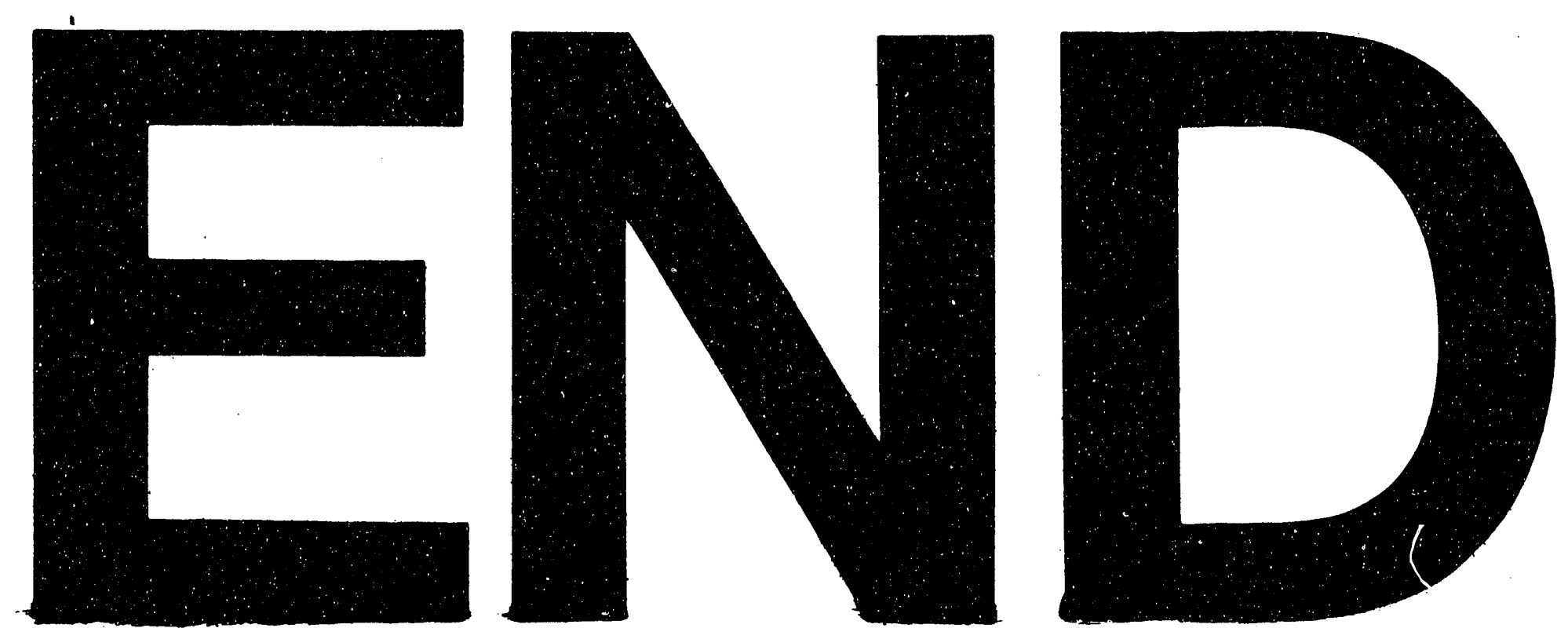

7

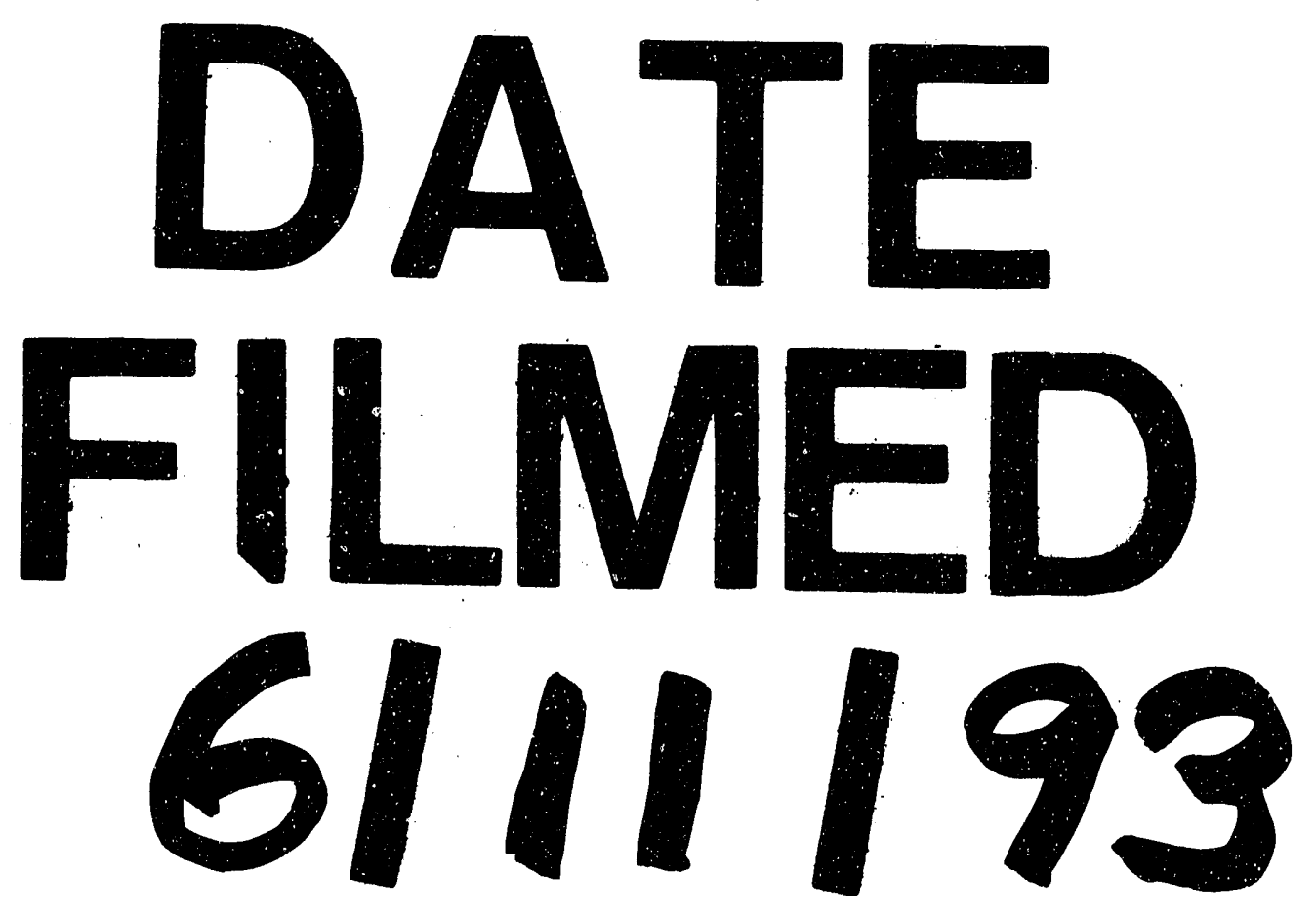




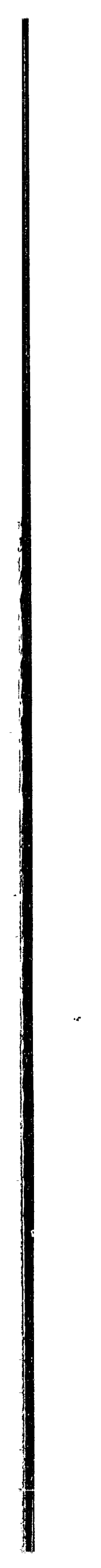

